\title{
La Universidad Popular José Martí en la órbita del pensamiento político de Julio Antonio Mella. Cuba, 1923-1927*
}

\section{YoEL CoRdoví NúÑEZ}

\begin{abstract}
Profesor del Instituto de Historia de Cuba y del Instituto Superior de Relaciones Internacionales (Cuba). Correo electrónico: ycordovi1971@gmail.com. El autor es doctor en Ciencias Históricas del Instituto de Historia de Cuba (Cuba). Entre sus publicaciones recientes tenemos: "Los manuales de higiene escolar para maestros en Cuba, 1902-1963”, en Historia y Memoria de la Educación No. 8 (2018) y en coautoría "Educación laica y enseñanza privada en Cuba. Principales proyectos, normativas y polémicas", Historia Caribe, Vol. 12 No. 30. Entre sus temas de interés se encuentran Historia de la educación en Cuba, Teoría y filosofía de la historia.
\end{abstract}

Recibido: 18 de enero de 2019

Aprobado: 19 de marzo de 2019

Modificado: 18 de junio de 2019

Artículo de investigación científica

DOI: https://doi.org/10.15648/hc.38.2021.2813

Este artículo forma parte del proyecto "La educación popular en Cuba, 1902-1961" financiado por el Instituto de Historia de Cuba.

Esta publicación está bajo una licencia Creative Commons Reconocimiento-NoComercial 4.0 (cc) BY-NC 
La Universidad Popular José Martí en la órbita del pensamiento político de Julio Antonio Mella. Cuba, 1923-1927

\section{Resumen}

El artículo tiene como objetivo principal determinar y analizar las etapas que marcaron los momentos de definición de la labor de la Universidad Popular José Martí, desde que fue fundada en La Habana en 1923 hasta su definitiva clausura por el gobierno de Gerardo Machado en 1927, así como valorar el alcance de sus objetivos y resultados. El texto se adentra en las actividades docentes y políticas de la Universidad Popular, concebidas en sus vínculos con el proceso de reforma universitaria, así como en la estructura administrativa, el diseño curricular de extensión y el significado de sus propuestas culturales y educativas contrahegemónicas. Estos problemas permiten comprender la estrategia política trazada por su artífice, el líder estudiantil Julio Antonio Mella.

Palabras clave: reforma universitaria, Universidad Popular, movimiento estudiantil, extensión universitaria, Julio Antonio Mella, Cuba, anti-imperialismo.

\section{People's University José Martí in the political thought orbit of Julio Antonio Mella. Cuba, 1923-1927}

\section{Abstract}

The main purpose of this article is to determine and analyze the stages indicating the definition moments of the role of People's University José Martí, from the time it was founded in Havana in 1923 until its final closure by Gerardo Machado government in 1927, as well as to consider the extent of its objectives and results. The text explores the teaching and political activities of this university, thought up in associations with the university reform process, as well as the administrative structure, the extension curricular design, and the meaning of its cultural and counter-hegemonic educational proposals. These problems allow the authors to understand the political strategy drawn up by its creator, the student leader Julio Antonio Mella.

Key words: university reform, People's University, student movement, university extension, Julio Antonio Mella, Cuba, Anti-imperialism.

\section{A Universidade Popular José Martí no âmbito do pensamento político de} Julio Antonio Mella. Cuba, 1923-1927

\section{Resumo}

O objetivo principal do artigo é determinar e analisar as etapas que marcaram os 
momentos de definição do trabalho da Universidade Popular José Martí, desde sua fundação em 1923 até seu encerramento definitivo pelo governo de Gerardo Machado em 1927, e analisar os objetivos e as conquistas da instituição. O texto explora as atividades pedagógicas e políticas da universidade, analisadas em seus vínculos com o processo de reforma universitária, bem como na estrutura administrativa no desenho curricular da extensão universitária e no significado de suas propostas culturais e educativas contra-hegemônicas. Estes problemas permitem compreender a estratégia política elaborada por seu inventor, o líder estudantil Julio Antonio Mella.

Palavras-chave: reforma universitária, Universidade Popular, movimento estudantil, extensão universitária, Julio Antonio Mella, Cuba, anti-imperialismo.

\section{L'Université Populaire José Martí dans l'orbite de la pensée politique de Julio Antonio Mella. Cuba, 1923-1927}

\section{Résumé}

L'objectif principal de cet article est celui de déterminer et analyser les étapes qui ont marqué les moments décisifs du travail de l'Université Populaire José Martí, fondée à La Havane en 1923, jusqu'à la fermeture définitive par le gouvernement de Gerardo Machado en 1927, et aussi d'évaluer la portée de ses objectifs et ses résultats. Le texte explore les activités pédagogiques et politiques de l'Université Populaire, conçues dans ses liens avec le processus de réforme universitaire, ainsi que dans la structure administrative, la conception curriculaire de l'extension universitaire et la signification de ses propositions culturelles et éducatives contre-hégémoniques. Ces problèmes permettent de comprendre la stratégie politique élaborée par son architecte, le leader étudiant Julio Antonio Mella.

Mots clés: réforme universitaire, Université Populaire, mouvement étudiant, extension universitaire, Julio Antonio Mella, Cuba, anti-impérialisme.

\section{INTRODUCCIÓN}

Apenas dos décadas después de inaugurada la República de Cuba, el 20 de mayo de 1902, se asistía a un inusitado proceso de expansión de movimientos sociales, representados por sectores y grupos más afectados por la crisis del modelo republicano neocolonial, insuflada 
tras la recesión mundial de los años 1920-1921‥ El desplome arrastró consigo a numerosos hacendados, colonos, comerciantes e instituciones bancarias, que vieron pasar sus propiedades a grandes consorcios industriales y financieros estadounidenses, pero las víctimas principales de la debacle estructural provenían de los estratos medios y pobres de la población. En ese contexto, una nueva generación de líderes obreros, intelectuales y estudiantiles, imbuidos por el impacto de eventos como la Revolución Mexicana, la Revolución Socialista en Rusia y la Reforma Universitaria de Córdoba, en Argentina, comenzaron a orientar sus agendas, con matices políticos e ideológicos diversos, hacia el enfrentamiento a los males sociales republicanos.

La franca orientación del gobierno de Alfredo Zayas (1921-1925) hacia la defensa de los intereses de los sectores agroexportadores cubanos en maridaje con la oligarquía financiera de Estados Unidos, los tratados arbitrarios y corruptos emprendidos por el cuarto presidente cubano, y el deterioro creciente de las condiciones de vida y trabajo de la población insular, condicionaron las más diversas manifestaciones opositoras al régimen ${ }^{2}$.

Entre los líderes que alcanzaban mayor grado de radicalización y madurez política se encontraba el estudiante de Derecho, Julio Antonio Mella (1903-1929). Hijo natural de un famoso sastre y comerciante cubano y nieto del prócer dominicano Ramón Mella, a escasos meses de matricular la carrera universitaria ya estaba involucrado en las principales protestas estudiantiles de la época ${ }^{3}$.

Pero las principales lides estudiantiles en las que se implicó Mella estarían por llegar. La autonomía universitaria prometida por Zayas y algunos congresistas había quedado solo en palabras con el beneplácito cias Sociales, 1992).

2 Julio Le Riverend, La república, dependencia y revolución (La Habana: Editora Universitaria, 1966). 3 En particular, fue de gran resonancia la protesta originada tras la propuesta de un grupo de profesionales al Consejo Universitario para que le fuera otorgado el título de Rector Honoris Causa al presidente Zayas y el doctorado, también honorífico, al general estadounidense Leonard Wood, segundo gobernador militar de la Isla (1899-1902), así como al entonces cónsul Enoch H. Crowder, representante de una política imperial abiertamente injerencista. 
del conservadurismo profesoral de la casa de altos estudios. Menos de un mes había transcurrido desde que el rector de la Universidad de Buenos Aires, el doctor José Arce, sostuviera un intercambio con el estudiantado universitario habanero, el 27 de noviembre de 1922, cuando los trabajos reformistas, ya en curso, se precipitaron. Con la constitución del Directorio de la Federación de Estudiantes de la Universidad de La Habana (FEU), presidido por Felio Marinello, y con Julio Antonio Mella como secretario, se intensificaron las exigencias de participación del estudiantado en el gobierno universitario, la depuración de su claustro y la autonomía total de la Universidad, considerada esta última base del resto de los cambios.

\section{Imagen No. 1}

Foto de Julio Antonio Mella realizada por su compañera Tina Modotti

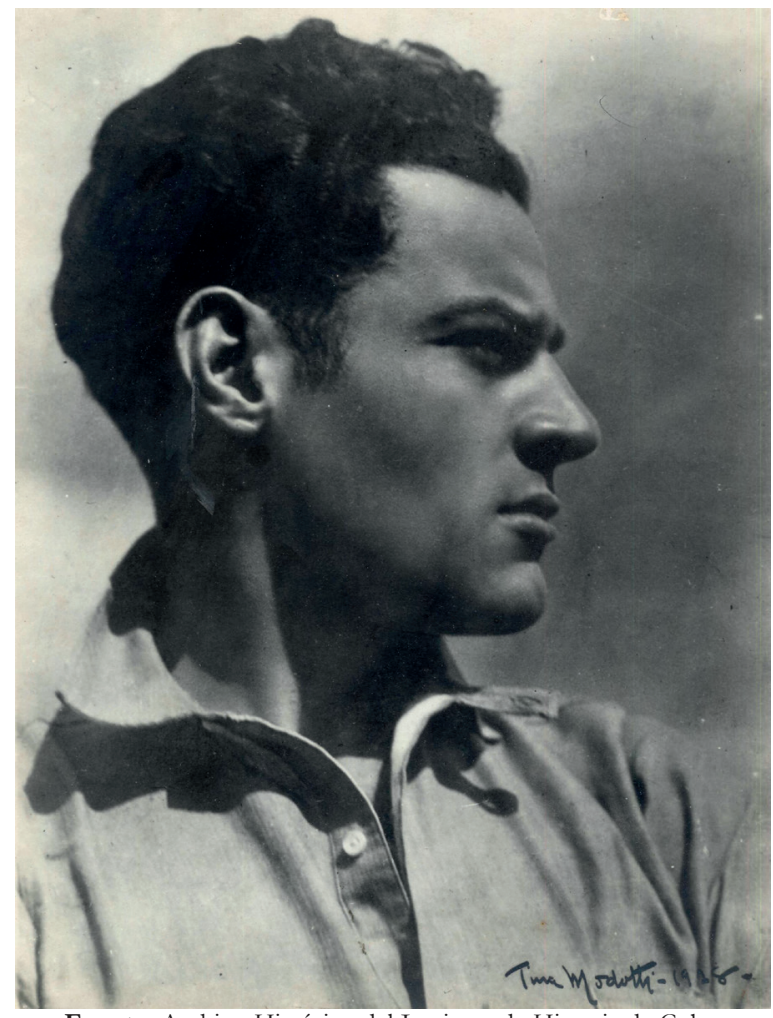

Fuente: Archivo Histórico del Instituto de Historia de Cuba.

Para Mella estaba clara la influencia en la Universidad habanera de la Reforma de Córdoba, irradiada a Chile, Uruguay y Perú en los inicios 
del movimiento, pero del mismo modo enfatizó la originalidad implícita en cada una de esas expresiones: "Lo original, lo prodigioso es que casi no ha obrado entre nosotros ninguna razón de contagio. Ha sido una aspiración común, de espontánea violencia, que la juventud americolatina ha sentido"4.

El presente artículo procura adentrarse en una de las instituciones de extensión educativa surgida al calor de la reforma universitaria, también como "aspiración común" que permeó el quehacer político de los dirigentes estudiantiles, obreros, profesionales e intelectuales de diferentes naciones de América Latina: la Universidad Popular José Martí (UPJM).

Desde luego, en modo alguno se trata de revelar una experiencia focalizada en el entorno cubano, sino su gestación y devenir como parte de un movimiento fundacional de universidades populares orientado a la educación de la clase obrera, tanto en América como en Europa desde finales del siglo XIX ${ }^{5}$.

¿Cómo surgió y en qué medida alcanzó perfiles propios en sus tareas, diferentes a las que le imprimiera la FEU a la reforma universitaria? ¿Qué lugar ocupó esta institución docente en la estrategia revolucionaria de Julio Antonio Mella? ¿Qué etapas pudieran demarcarse en los cuatro años de existencia de la Universidad Popular?, he aquí algunas de las interrogantes a dilucidar. La consulta de las obras de Mella, compiladas en diferentes ediciones, los testimonios publicados de profesores y activistas de la UPJM, así como el procesamiento del fondo Partido Marxista Leninista, existente en el Archivo Histórico del Instituto de Historia de Cuba, permitirán enrumbar la investigación alrededor de los problemas expuestos.

"Hablando con Julio Antonio Mella sobre la revolución universitaria", La Habana, 23 de noviembre de 1924, en Julio Antonio Mella. Documentos y artículos, ed. Instituto de Historia del Movimiento Comunista y la Revolución Socialista de Cuba (La Habana: Editorial de Ciencias Sociales, 1975), 133.

5 Las universidades populares surgieron en Francia a finales del siglo XIX, asociadas a la iniciativa del obrero autodidacta Georges Deherme (1867-1937). En marzo de 1899 se constituyó la Sociedad de las Universidades Populares, orientada a lograr la propagación de esas entidades por toda Francia. En el caso de España fue Vicente Blasco Ibáñez (1867-1928) el fundador de la primera Universidad Popular en su Valencia natal en 1903. Vid. Pedro Luis Moreno Martínez, "Haciendo memoria: las Universidades Populares en España”, Tabanque. Revista Pedagógica No. 19 (2005): 26-27. 


\section{Julio Antonio Mella. De la reforma universitaria al Congreso Nacional de Estudiantes}

El ascenso del liderazgo estudiantil de Mella entre la marea del reformismo universitario, coincidía con sus inclinaciones por las luchas sindicalistas. Alternaría las reuniones del Directorio de la FEU con las visitas al Centro Obrero de Zulueta No. 37 y la Sociedad de Torcedores de La Habana. De esos contactos surgió el interés de que sus dirigentes participaran en la asamblea estudiantil en apoyo a la solicitud de los alumnos de quinto año de la carrera de Medicina consistente en la modificación de los estatutos de la Universidad. Mientras esos reclamos no se atendieran, se recomendaba a todos los estudiantes abstenerse de asistir a clases. Al acto asistió una representación obrera, cuyos principales voceros publicitarios, Justicia, Nueva Luz y Boletin del Torcedor, se hicieron eco del alcance del reformismo universitario: "No es en el campo del proletariado solamente que ha germinado la semilla regada por Ingenieros, Barbusse, Anatole de France y tantos otros, también en las aulas universitarias, en el campo de la intelectualidad; florecen, de acuerdo con los tiempos nuevos, ideas nuevas".

La otra fuerza importante de apoyo a la manifestación del estudiantado universitario fueron los alumnos de los institutos provinciales, escuelas normales para maestros, escuelas de Artes y Oficios, además de los colegios y academias, tanto laicos como religiosos.

Estudiantes y obreros, he aquí los pilares de la estrategia revolucionaria de Mella. Era una alianza que presuponía la misión revolucionaria de los estudiantes identificados con las necesarias transformaciones estructurales que requería el país. El alumnado universitario, el de la enseñanza media y el de otros niveles tendría la tarea de vincularse a la educación popular, insuflar su magisterio en escuelas y academias nocturnas, algunas establecidas desde inicios del siglo XX, pero con presupuestos marcadamente anticapitalistas. Al mismo tiempo, estaba consciente Mella que ese desbordar el recinto universitario de la mano de estudiantes y obreros implicaba una garantía para la continuidad de

6 Justicia, La Habana, 13 de enero de 1923, 1 
la lucha universitaria y social, a sabiendas de que el escenario físico que ocupaba la Universidad Nacional, donde inició él su quehacer reformista, se hacía cada vez más peligroso.

Explicable lo difícil de investir a la reforma universitaria de un sesgo tan radical, máxime por un líder carismático que llegaba a la palestra pública enfrentando a Rectores, a Secretarios de Instrucción Pública y a Presidentes de la República. La reacción de quienes se oponían a Mella, entre los que se encontraban estudiantes de ideas conservadoras, no se haría esperar. Era necesario disponer de otro escenario donde convergieran, a modo de depuración, aquellos jóvenes que abrazaban las ideas de transformación más radicales. Mella así lo entendió. De ahí su protagonismo en la organización y dirección del Primer Congreso Nacional de Estudiantes, celebrado en La Habana entre los días 15 y 25 de octubre de 1923.

La profunda distancia de contenidos entre la reforma universitaria y el congreso estudiantil fue advertida por el historiador y testigo de la época Sergio Aguirre, cuando expuso que la reforma universitaria "fue un hecho docente, de forzosa inmadurez inicial, con el cual se quiso, un tanto candorosamente, sanear la rama podrida -la universidad- de un tronco podrido -la república neocolonial". El Congreso de Estudiantes, en cambio, "fue un hecho político, medularmente político, que utilizó como elemento de atracción para la masa estudiantil los prestigios alcanzados por la Reforma Universitaria"7.

En la Declaración de los Derechos y Deberes del Estudiante, propuesta por Mella y aprobada por unanimidad en el Congreso, se hallaban las principales premisas de la reforma universitaria. No obstante, se avanzaba en otros temas que rebasaban el ámbito docente y administrativo de la Universidad, en particular cuando se refería al deber de los estudiantes de divulgar sus conocimientos en la sociedad, principalmente entre los obreros manuales. No sería casual que, entre los acuerdos tomados en el cónclave juvenil, apareciera el estrechamiento de las relaciones entre el estudiantado con la Federación Obrera de La Habana (FOH), consti-

7 Sergio Aguirre, “Un toque de clarín en 1923”, Bohemia, La Habana, 19 de octubre de 1973, 100. 
tuida por el presidente de la Asociación de Tipógrafos, el sindicalista Alfredo López, a finales de 1920. Ahora bien, como parte de esa labor de extensión, imposible de lograr en los marcos de la Universidad de La Habana, se aprobaría la creación de una Universidad Popular que llevaría por nombre "José Martí", ideólogo revolucionario e iniciador de la revolución independentista de 1895.

Imagen No. 2

Primer Congreso Nacional de Estudiantes celebrado en el Aula Magna de la Universidad de La Habana, 15-25 de octubre de 1923

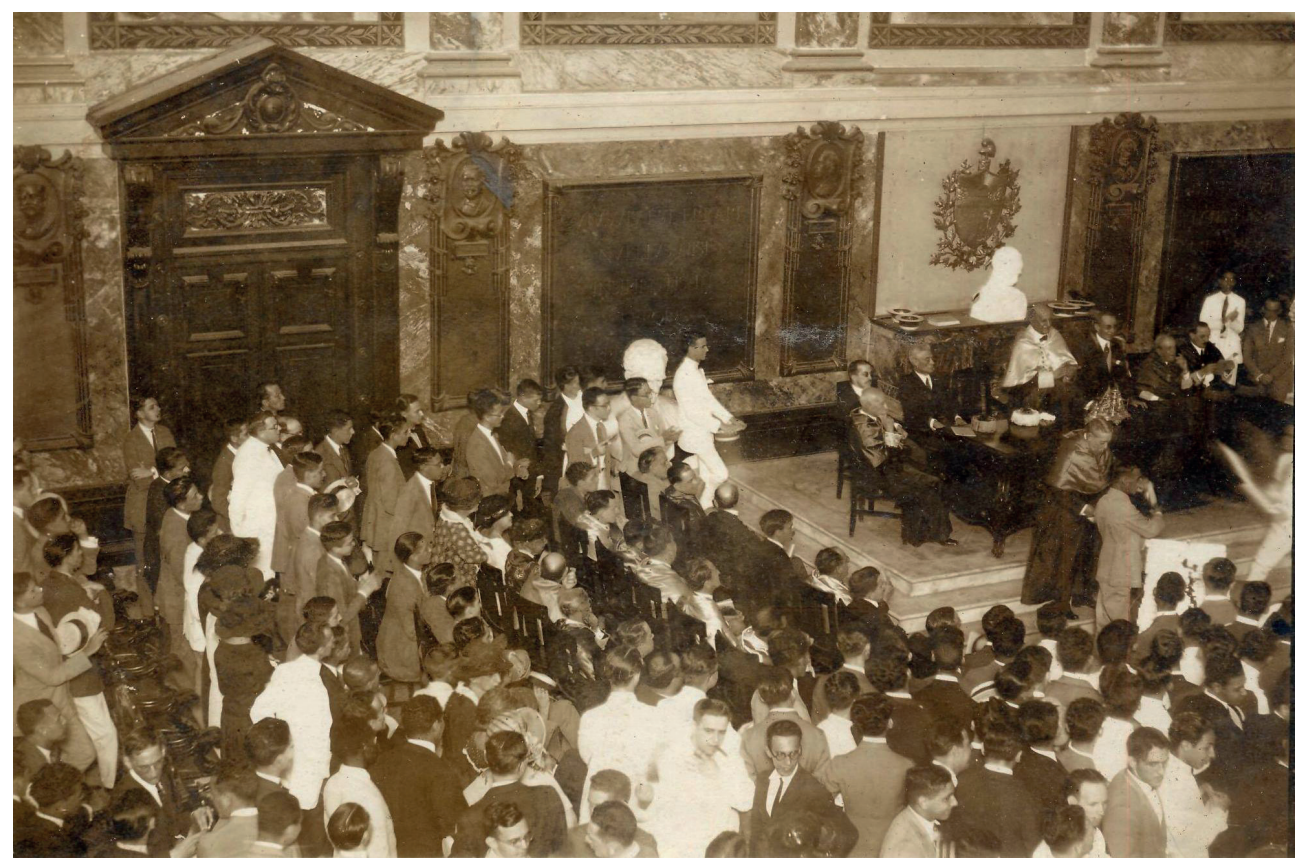

Fuente: Archivo Histórico del Instituto de Historia de Cuba.

\section{La Universidad Popular José Martí en la estrategia POLÍTICA DE MELLA}

El 3 de noviembre de 1923 quedaron aprobados los ocho estatutos constitucionales de la UPJM. En síntesis, el funcionamiento de la universidad proletaria, como la definió Mella, partiría de un conjunto de premisas, algunas sustentadas por una tradición de pensamiento "antidogmático y científico", al que se le incorporaba la lucha por la justicia social, componente nuevo, resultado de un pensamiento revolucionario que comenzaba a articularse y definir sus contornos en la 
década del veinte del pasado siglo. De tal suerte, la UPJM, procuraría "formar en la clase obrera de Cuba y en cuanto acudan a sus aulas, una mentalidad culta, completamente nueva y revolucionaria".

Era un modo de redimensionar la acción estudiantil, hacerla extensible más allá del reducido marco docente y administrativo universitario, de forma tal que pudiera ubicarse en el centro de los problemas que aquejaban a la nación cubana.

La tarea en modo alguno era sencilla. Mella y sus seguidores tenían por delante el complejo escenario de tendencias políticas obreristas gravitando sobre un movimiento obrero fraccionado y con marcada presencia del ideario y las prácticas anarcosindicalistas y reformistas. A este cuadro se añadían los trabajos de dirigentes reformistas de la Federación Tabacalera y de la Federación Obrera de la Bahía de La Habana para facilitar la penetración de la Confederación Obrera Panamericana (COPA) en las organizaciones obreras, un primer paso para adherirlas a la American Federation of Labour (AFL), suerte de agencia sindical de Estados Unidos en América Latina al servicio de las patronales y los consorcios imperialistas.

Ciertamente, del seno obrero habían emergido algunas experiencias de educación popular, pero sin que estas llegaran a consolidarse. Destacable en aquel escenario fue la impartición de conferencias por prestigiosas personalidades de la cultura cubana en el Centro Obrero, institución fundadora de una escuela para adultos en 1923, llamada a desvanecerse debido a la carencia de personal docente. No mejor suerte alcanzó la primera Escuela Racionalista, fundada ese mismo año por el líder comunista de origen canario José Miguel Pérez, dirigida a formar a los hijos de obreros en una ideología marxista.

La UPJM, en tanto experiencia formadora de una cultura marxista desde la base, con la unión de estudiantes, profesionales e intelectuales, estaría en mejores condiciones de alcanzar tales propósitos.

8 Julio Antonio Mella, "Estatutos de la Universidad Popular José Martí", 3 de noviembre de 1923, en Mella. Textos escogidos, comp. Julio César Guanche, t. I (La Habana: Ediciones La Memoria, Centro Cultural Pablo de la Torriente Brau, 2003), 104. 
Una primera etapa en el quehacer de la UPJM se inició el 20 de diciembre de 1923, cuando se inauguraron sus primeros cursos en la Facultad de Derecho de la Universidad de La Habana. La enseñanza tenía diferentes niveles: los de primera enseñanza que comprendían las escuelas de analfabetos y la nacional. Para la segunda enseñanza el plan de estudio establecía las asignaturas Geografía Universal, Historia de la Humanidad y de Cuba, Psicología y Lógica, Gramática y Literatura, Cívica, Historia Natural, Estudios Generales (Medicina de Urgencia e Higiene), Homicultura ${ }^{9}$, Maternidad y Profilaxis Sexual, Ciencias Naturales, Economía Política y Social, Derecho, Legislación del Trabajo, Moral Antidogmática y Rudimentos de Ciencias de las Religiones. Las asignaturas de Matemáticas, Física y Química no lograron cubrirse con profesores, al menos en el plan inicial ${ }^{10}$.

No sucedió así con las conferencias sobre política internacional y de divulgación artística, literaria y científica, impartidas por renombradas figuras de la intelectualidad en institutos, escuelas normales, escuelas de Artes y Oficios, Liceos, Centros Regionales, Sociedades Deportivas, etc. Al llamado de los organizadores acudieron conferencistas de la talla del historiador Emilio Roig de Leuchsenring, Fernández de Castro, Luis A. Baralt, Arturo Montori y Alfredo M. Aguayo. Entre los estudiantes y graduados que participaban junto con Mella se encontraban los militantes comunistas Sarah Pascual, fundadora de la Liga Juvenil Comunista, y Leonardo Fernández Sánchez, además de prestigiosos abogados, médicos y psicólogos, como Alfonso Bernal del Riesgo, Eusebio A. Hernández y Bernardo Valdés Hernández.

9 Término introducido por el profesor de Obstetricia y Ginecología de la Universidad de La Habana, el Dr. Eusebio A. Hernández, junto con el Dr. Domingo F. Ramos, al considerarlo más apropiado que el de "Puericultura", especialidad dedicada al estudio, conservación, desarrollo y mejoramiento de la especie humana. La Homicultura, en su concepción más integral, tendría en cuenta no solo la atención a la madre gestante, sino también medidas de carácter eugenésico como la "profilaxis sexual", entre las que se encontraba el certificado médico prenupcial. Vid. Eusebio Hernández y Domingo Ramos, "Homicultura", Boletín de Sanidad y Beneficencia T. VI, No. 1-6 (La Habana, 1911).

10 "Plan de estudios y profesores de la Universidad Popular José Martí", en Mella. 100 años, ed. Ana Cairo, Vol. I (Santiago de Cuba: Editorial Oriente, 2003), 51-52. 
Imagen No. 3

Profesores que integraron el claustro de la Universidad Popular José Martí, entre los que se encuentra Mella.

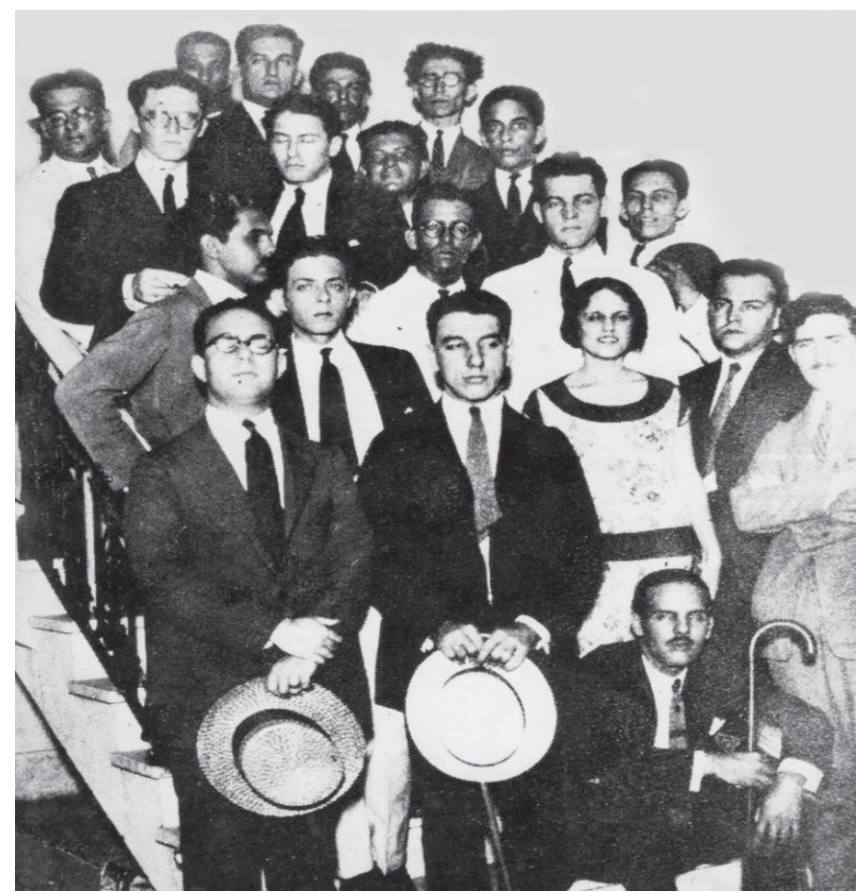

Fuente: Archivo Histórico del Instituto de Historia de Cuba

Cada noche, aquel grupo de estudiantes e intelectuales revolucionarios, según Roa, derramaba su fervor y la luz de la teoría marxista ante un auditorio estremecido y compacto ${ }^{11}$, donde se agrupaban más de 500 obreros de diferentes sindicatos ${ }^{12}$. En el grupo pronto figurarían otros destacados jóvenes revolucionarios como el médico Gustavo Aldereguía Lima, el poeta y periodista José Zacarías Tallet y el intelectual Rubén M. Villena.

De extraordinaria importancia fue la presencia de Martínez Villena en el claustro universitario. El joven intelectual, doctor en Derecho Civil y Público, estaba ganado para la lucha revolucionaria, luego de prota-

11 Raúl Roa, "Una semilla en el surco de fuego", en Órbita de Rubén Martínez Villena, selección de Roberto Fernández Retamar (La Habana: Colección Órbita, UNEAC, 1964), 41.

12 Sarah Pascual, Julio Antonio Mella. Síntesis de su vida (La Habana: Universidad de La Habana, Instituto Julio Antonio Mella, 1971). 
gonizar la enérgica Protesta de los Trece, primera acción de protesta pública contra la corrupción política-administrativa en Cuba, y de fundar la Falange de Acción Cubana. Cuando conoció a Mella, justo en los trabajos preparatorios del Primer Congreso de Estudiantes, tenía un historial reconocido, no solo en el mundo de la literatura, sino también de la política. Arrastraba, de hecho, las memorias del presidio tanto en cárceles de Cuba como de Estados Unidos.

El otro hecho significativo tanto en la integración del claustro de la Universidad Popular como en sus orientaciones políticas fue la llegada de varios estudiantes deportados de Venezuela y Perú, víctimas de las dictaduras de Juan Vicente Gómez y Augusto Bernardo Leguía, respectivamente. Al decir de uno de sus más prestigiosos profesores, "Salvador de la Plaza y Gustavo Machado, y Luis F. Bustamante y Jacobo Hurwitz, traían encendidos el fuego de la nueva inquietud y la carne joven ya macerada por el hierro opresor"13.

La cantera selecta de la UPJM habría de engrosarse cuando en febrero de 1924, Mella fundó el Ateneo Universitario José Martí, bajo el lema "Crear hombres cultos con ideología renovadora". La misión principal de la institución sería apoyar el trabajo de la Universidad Popular. Ese mismo año fundó otras dos agrupaciones importantes cuyos integrantes tendrían vínculos con la Universidad: la Federación Anticlerical de Cuba y la Confederación de Estudiantes de Cuba, ambas presididas por Julio Antonio. A mediados del año siguiente se añadiría la sección cubana de la Liga Antimperialista de las Américas, entre las asociaciones de mayor fuerza en el sostén ideológico de la UPJM y en un sentido más amplio de toda la proyección estratégica del líder con base en la educación para la transformación social.

De esos elementos se rodearía Mella para una tarea que trascendía la inmediatez de cualquier acto de reforma. Consciente del escenario cada vez más hostil, ya no solo en el logro de la autonomía universitaria, sino para la continuidad de los objetivos alcanzados, buscó nuclear una

13 Raúl Roa, "Una semilla en el surco de fuego", 42. 
vanguardia de jóvenes de ideas radicales. El primer paso consistió en presentar su renuncia del cargo de presidente de la FEU a finales de 1923. Desde luego, como bien apreciara la historiadora cubana Olga Cabrera, la dimisión del líder estudiantil no significó su alejamiento de las actividades estudiantiles, sino un cambio de táctica, muy a tono con su decisión de aislarse de sus seguidores o adversarios timoratos y plantear su estrategia de lucha en un terreno y con prosélitos mucho más favorables. Con claridad meridiana, expuso estas ideas al médico argentino Gregorio Araoz Alfaro:

“Tú no sabes los enemigos que tiene, por lo menos en este país, el movimiento regenerador universitario, los mayores enemigos son los mismos estudiantes faltos de ideologías o envenenados con la ideología reaccionaria de los colegios religiosos. Estos forman gran mayoría en nuestra Universidad.

Así y todo, se hará lo que se pueda. Yo tengo la firme convicción que siempre es bueno agitar e intentar, a cada momento, una renovación. Con obtener la creación o el descubrimiento de muchachos con inquietudes y rebeldías creo que se hace un gran beneficio social, por el momento" $"$.

Muchos de sus aliados en el claustro, esos "muchachos con inquietudes y rebeldías", eran estudiantes que integraban el grupo Renovación, otros habían contactado con la Agrupación Comunista de La Habana. Por la parte de Mella se producía el estrechamiento de relaciones con la FOH y con el profesorado y el estudiantado más progresista de los institutos de segunda enseñanza en las diferentes provincias del país.

Algunos autores, incluso, han expuesto las implicaciones del cambio de nombre del órgano periodístico estudiantil Alma Mater por Juventud, a finales de 1923. Según Cabrera, era la expresión de una concepción diferente acerca del papel de los jóvenes en la lucha social: "Juventud representaba a los estudiantes renovadores, a la vanguardia de los

14 Julio A. Mella, "Carta a Araoz Alfaro", Camagüey, 3 de enero de 1924, en Hasta que llegue el tiempo, eds. Adys Cupull y Froilán González (La Habana: Editora Política, 2002), 197. 
jóvenes"15. La historiadora alemana Christine Hatzky coincide en ese enfoque cuando advierte que Juventud "reflejaba los criterios y las discusiones del ala más radical de los estudiantes, una vanguardia intelectual y política que solo representaba a una minoría de los estudiantes"16.

De inmediato el rumbo nacionalista de la pléyade de estudiantes alcanzó una orientación antimperialista y latinoamericanista, plasmada en los editoriales de Juventud y la revista Venezuela Libre, esta última liderada por Villena, en tanto foros de discusión, de denuncias, pero también de instrucción. Hatzky valora el influjo de las obras literarias, culturales y filosóficas de José Ingenieros, Anatole France, Henri Barbusse, Máximo Gorki, José Vasconcelos y los cubanos Enrique José Varona y Carlos Baliño en las páginas de Juventud.

Y en torno a esa tribuna publicitaria se hallaba el núcleo rector que convergía con Mella en la UPJM. Su concepción de la reforma universitaria deslindaba bien las fronteras, valiéndose de una retórica osada, extremadamente radical como para ser aceptada por los espíritus reformistas, ni siquiera por muchos de los que preferían calificarla de "revolución". En esa demarcación quedaba establecida, por decantación, la misión de la Universidad Popular en Cuba: "Pero si la reforma va a acometerse con seriedad y con espíritu revolucionario no puede ser acometida más que con un espíritu socialista, el único espíritu revolucionario del momento" 17 .

A tres años de aprobarse el nuevo plan de estudio de la república (19211926) por la Secretaría de Instrucción Pública y Bellas Artes, el líder estudiantil entiende que existían obstáculos gigantescos que obstaban el acceso al sistema educativo de los sectores y grupo más vulnerables a la pobreza; el principal provenía de la misma penuria de gran parte de la población en edad escolar, pero también de la inexistencia de

15 Olga Cabrera, Los que viven por sus manos (La Habana: Editorial de Ciencias Sociales, 1985), 330.

16 Christine Hatzky, Julio Antonio Mella. Una biografía (Santiago de Cuba: Editorial Oriente, 2008), 131.

17 Julio Antonio Mella, "El concepto socialista de la reforma universitaria", El Tren Blindado, México (s/f), Archivo Histórico del Instituto de Historia de Cuba (AHIHC), Fondo Primer Partido Marxista-Leninista, 1/2: PE 2.2/7/63. A1/1-15. 
una política de educación popular. Por otra parte, está consciente de la naturaleza y los objetivos adaptativos de los planes y cursos de estudio estatuidos por las instancias estatales, a tono con los intereses de las clases sociales en el poder. De ahí que la "primera escuela proletaria de Cuba", de acuerdo con Mella, si bien programaba la cultura entre obreros y estudiantes, no era "la cultura hipócrita y oficial" al uso. Más bien se trataba de destruir "una parte de las tiranías de la actual sociedad: el monopolio de la cultura".

"Nosotros llevamos hasta las clases populares, hasta los obreros gremiales, un saber complejo y dúctil, generalizado, consciente. Comprende desde los altos cursos, de naturaleza superior, hasta lo más elemental, que es el propio alfabeto. Hacemos, por lo tanto, un ensayo práctico de nuestra teoría constructiva. Nos mueve un plan"18.

He aquí, en palabras de Mella, la esencia de la UPJM como instrumento transformador y gestor de una cultura contrahegemónica capaz de movilizar con vistas a operar el cambio social necesario. Asistimos así a la ruptura epistemológica de la década del 20, a la que alude el escritor argentino Néstor Kohan, cuando se refiere a la transición de un "marxismo en América Latina" a un "marxismo de América Latina", conversión a la que Mella aporta de manera indudable:

"Se superan continentalmente los límites económicos-corporativos del movimiento socialista para plantearse por primera vez el desafío hegemónico -específicamente político, ideológico y cultural- de formular tareas para el conjunto social, tareas que en nuestro continente deben tejer y enhebrar con un mismo hilo las luchas clasistas, proletarias y campesinas con las antimperialistas (y nacionales)"19.

Con razón J.C. Guanche afirma que "de la ciudad letrada, Mella es el ciudadano incómodo"20, sabe que la virtud cívica es condescendiente con el orden, y aún el mejor de los ciudadanos respondería en aquel

20 Julio César Guanche, “Julio Antonio, qué pasa en Cuba?”, en Mella. 100 años, Vol. II, 345. 
contexto a la preservación decente de un orden irremediablemente capitalista. Otra debía ser la racionalidad de la enseñanza en el país y cifró en la labor de extensión universitaria las posibilidades de preparar culturalmente a los obreros, de concientizar a quienes estaban llamados a subvertir el orden por medio de una revolución social, dentro y fuera de Cuba, a tono con los postulados de "frente obrero único", fórmula enarbolada en el tercer y cuarto congresos de la III Internacional Comunista, celebrados en 1921 y 1922, respectivamente.

Era la táctica del trabajo partidario, aplicada consecuentemente por los partidos marxistas en América Latina, que cifraba la toma del poder político por el proletariado a partir de la movilización de amplios sectores y grupos de trabajadores, fuesen o no integrantes de la clase obrera. Mella, junto con el peruano José Carlos Mariátegui y el chileno Luis Emilio Recabarren, se encontraba entre los portadores más influyentes de este ideario político en la región.

Desde luego, era imposible que tales orientaciones motivaran la concepción de los planes de estudio oficiales. De aprobarse la autonomía total universitaria en Cuba las posibilidades de incidir en el diseño curricular de los cursos de extensión desde dentro de la propia Universidad, sin requerir de la aprobación del gobierno, quizá podía abrir un margen mayor de acción radical. Eso lo sabía Mella, pero también los funcionarios del gobierno.

El trabajo se haría más difícil, pero para ello Mella disponía de jóvenes radicales que lo seguían en la consecución de una educación popular, tan difundida por el intelectual argentino José Ingenieros, a quien el dirigente cubano conociera personalmente en La Habana, en 1925. Además, la socialización del conocimiento entre las capas populares contaba con experiencias importantes hacia esa fecha: las campañas de alfabetización desarrolladas por Anatoli Lunacharsky, Comisario de Instrucción Pública de Rusia, y la reforma educativa implementada por José Vasconcelos, Secretario de Instrucción Pública en México, que incluía las escuelas rurales y las misiones culturales. 
De Europa llegaban otras iniciativas con mayor o menor influencia, al estilo de la Escuela Marxista de París; las escuelas del Partido Laborista Independiente de Inglaterra, en las que se destacaba el magisterio del catedrático de Cambridge, Bertrand Russell; la Universidad Popular de Milán y la Universidad Obrera de Varsovia.

Pero sin duda fueron las Universidades Populares latinoamericanas las de mayor influjo en Cuba, en particular la Manuel González Prada, fundada por la Federación de Estudiantes del Perú, en el marco del Primer Congreso de Estudiantes en Cuzco, a inicios de 1921. La Universidad Popular peruana contó en Mella con un fiel defensor, de ahí su repulsa ante el encarcelamiento de uno de sus profesores más influyentes en América Latina: el intelectual marxista José Carlos Mariátegui. En carta al representante diplomático del Perú en Cuba, a nombre de los obreros y estudiantes de la UPJM, el líder cubano expresaba su indignación por los atropellos del dictador Leguía contra la institución popular andina, en la persona de Mariátegui, quien fuera confinado, según Mella, "por el solo delito de pensar libremente"21. Del Amauta peruano son estas consideraciones que Mella incorporó al Boletín de la UPJM:

"Las universidades populares no son institutos de agnóstica e incolora extensión universitaria. No son escuelas nocturnas para obreros. Son escuelas de cultura revolucionaria. Son escuelas de clase. No viven adosadas a las academias oficiales ni alimentadas de limosnas del Estado. Viven del calor y de la savia populares. No existen para la simple digestión rudimentaria de la cultura burguesa. Existen para la elaboración y la creación de la cultura proletaria"22.

Imposible descartar la influencia que ejerció en una primera etapa, tanto en la reforma universitaria cubana como en la fundación de la UPJM, la ejecutoria del líder peruano Víctor Raúl Haya de la Torre, organizador del referido Congreso de Estudiantes en el país austral y fundador de la

21 Julio Antonio Mella, "Carta al representante del Perú", mayo de 1924, en Mella. 100 años, Vol. II, 175.

22 José Carlos Mariátegui, "Las Universidades Populares”, Boletín de la Universidad Popular José Martí, La Habana, 13 de julio de 1924, 2. 
Alianza Popular Revolucionaria Americana (APRA), por más que luego encontrara en Mella a uno de sus más agudos críticos.

\section{Más allá de la colina: la Universidad Popular José Martí EN SUS NUEVAS SEDES}

A partir del 3 de noviembre de 1924 pudiera delimitarse una segunda etapa en la vida de esta institución, marcada por su salida de los predios universitarios. Ese día las clases dejaron de impartirse en la Facultad de Derecho de la colina universitaria para trasladarse al local de la Federación Anticlerical de Cuba, ubicada en la calle Reina No. 72, así como a las sucursales de los barrios de Luyanó y Príncipe. El cambio de sede era muestra de la avanzada contrarreformista y el engrosamiento de la FEU por quienes Mella denominó “jóvenes sensatos” y de "orden", partidarios de mantener intacto el tablero de la política universitaria y de la nación. La misma dinámica favorecería la apertura de filiales fuera de La Habana, como la de San Antonio de los Baños, Santiago de las Vegas, Regla y Guanabacoa. En un balance hecho por Mella en el primer año de trabajo de la UPJM, salían a relucir algunos de los principales escollos enfrentados:

"En el pasado curso tuvimos que luchar con múltiples dificultades. El efervescente temperamento tropical hizo que esta labor en los comienzos tuviera muchos adeptos, pero pronto lo arduo de la obra nos trajo las primeras bajas.

La reacción entronizada más tarde en la Universidad, el fracaso ruidoso de la Federación de Estudiantes -su casi disolución- al caer este organismo en manos de los jóvenes "sensatos", "de orden", etc., al abandonarlo el elemento renovador que realizó la Reforma Universitaria, por los cobardes temores de los jóvenes viejos y las intrigas y ambiciones de los mediocres que aspiraban a medrar, fueron las principales causas de la crisis por que atravesó la Universidad Popular José Martí.

Un grupo de estudiantes verdaderamente idealistas sostuvieron la bandera de la cultura revolucionaria. Las huestes obreras permanecieron fieles a sus deseos de mejorarse, de emancipación del dominio de la cultura y el Primero de Mayo, cuando se clausuró el curso, eran 
profesores y alumnos la mitad de los que comenzaron la sublime labor en noviembre; pero esa mitad era sincera y llena de fe en el triunfo de la campaña. Por eso era fuerte.

Ahora abrimos el Curso de verano el domingo 13 de julio y la Universidad Popular surge más potente y más prometedora"23.

No obstante, el hecho de que traspasara los muros que albergaban a la oposición, posibilitó también que estudiantes y profesores de la UPJM gozaran de un margen de libertad mayor, sobre todo para el trabajo político. Esta sería la etapa de mayor activismo de la institución de educación popular. Incluso algunas de las iniciativas tendieron a incidir en los cambios desde afuera hacia adentro, es decir, procuraban introducir en la Universidad Nacional de la reacción o la "universidad vieja", como la calificara Mella, los ideales revolucionarios de la UPJM. Pudiera citarse el manifiesto redactado por el ejecutivo de la universidad proletaria dirigido a obreros, intelectuales y estudiantes, incluidos los matriculados en la Universidad de La Habana, en apoyo a las demandas de los sindicatos en los centrales azucareros: "La Universidad Popular 'José Martí pide a las asociaciones estudiantiles y culturales que dejen oír por los periódicos o manifiestos su voz de protesta ante los atropellos del gobierno y del imperialismo yanqui" ${ }^{24}$.

En marzo de 1925, Mella gestionó la instalación de sus aulas en el Instituto de Segunda Enseñanza de La Habana, donde había cursado sus primeros estudios de Bachiller y en cuyo local de la Asociación de Estudiantes del Instituto de La Habana contaba con el apoyo de su incondicional compañero de lucha Leonardo Fernández Sánchez. Para el nuevo curso Mella presentó una serie de conferencias sobre problemas de actualidad nacional: I- El fracaso del sistema político, IILa traición de los intelectuales, III- El fracaso de la Revolución Universitaria, IV- El peligro del capitalismo yanqui. Asimismo, impartía las clases de Legislación Obrera, Historia y otras disciplinas ${ }^{25}$.

23 Julio Antonio Mella, "A los alumnos de la Universidad Popular y al pueblo de Cuba", 3 de agosto de 1924, en Julio Antonio Mella. Documentos y artículos, 100-101.

24 El Heraldo, La Habana, 26 de noviembre de 1924.

25 Erasmo Dumpierre, Julio Antonio Mella. Biografía (La Habana: Editorial Orbe, 1975), 73. 
A las clases nocturnas se incorporaron las conferencias dominicales con temas sociales, impartidas en el cine Wilson, centro cultural ubicado entre las transitadas calles Belascoaín y San Rafael. Al decir de Padrón, uno de los líderes obreros de la época:
"Con estas conferencias Julio Antonio Mella transmitía a los oyentes sus conceptos antimperialistas, y a la vez, despertaba en ellos una conciencia revolucionaria de clase, para liberarlos del marasmo mental creado por las ideas del apoliticismo y el nihilismo anarquista y la de colaboración de clases del reformismo"26.

También las carteleras de cine de la UPJM, localizadas en el Archivo del Instituto de Historia de Cuba, anunciaban las proyecciones de películas y otras actividades culturales, como cantos, comedias y exposiciones $\operatorname{artísticas~}^{27}$. El empleo de la cinematografía y del teatro se expandiría a partir del derrocamiento de la dictadura machadista, en 1933, mediante las misiones culturales, a cargo de instituciones del Estado, en particular la Dirección de Cultura de la Secretaría de Instrucción Pública y Bellas Artes y del Consejo Corporativo de Salubridad y Beneficencia, solo que con un radio de acción y basamentos político-educativos diferentes.

Otras iniciativas que, aunque no lograron materializarse, mostraron el interés de la dirección de la Universidad Popular por extender la educación, fueron los proyectos de bibliotecas públicas.

En el terreno de la política, el Comité Ejecutivo de la UPJM promovió iniciativas diversas, como la convocatoria a la conmemoración del Día Internacional de los Trabajadores y la consecuente formación de un Comité Conjunto Pro Primero de Mayo.

Imposible desligar la agenda de la Universidad Popular del liderazgo político de su fundador. Con solo 22 años, fundó la Liga Antimperialista de Cuba y asistió al Primer Congreso Nacional de Agrupaciones Comunistas de Cuba los días 15 y 16 de agosto de 1925, hecho que

26 Pedro Luis Padrón, Julio Antonio Mella y el movimiento obrero (La Habana: Editorial de Ciencias Sociales, 1980), 81.

27 AHIHC, Fondo Primer Partido Marxista-Leninista, 1/2: PE 2.1/1/1/1. 
culminaría con la fundación del Partido Comunista de Cuba (PCC). Ese mismo mes surgía la Confederación Nacional Obrera de Cuba (CNOC), central sindical que mostraba el ascenso vertiginoso del movimiento obrero en el decurso de los cinco primeros años de la década de 1920. Es decir, se asistía a la emergencia de alternativas marxistas de organización popular para enfrentar la crisis del modelo republicano y las variantes reaccionarias de la oligarquía azucarera, con su principal carta de triunfo en la silla presidencial: el general Gerardo Machado Morales (1925-1933)

Desde la llegada al poder, Machado se enfrascó en eliminar a sus principales opositores $^{28}$. El joven Mella estaba entre ellos y, con él los principales instrumentos de organización política implementados para preparar las condiciones que llevarían al estallido de la revolución social en Cuba. La mirada del dictador pronto se dirigió a la UPJM.

\section{LA CONTRARREFORMA UNIVERSITARIA Y LA CLAUSURA DE LA UPJM}

Hacia 1925, la contrarreforma universitaria ganaba espacios importantes. Un testigo excepcional de los hechos se refería al modo en que los adversarios proliferaban en el Consejo Universitario y en el claustro: "No podía ser de otra manera, dada la composición social de la Universidad, la estructura económica de la república, la formación escolástica de la juventud, el desbarajuste imperante y el complejo de inferioridad colonial. Aún los tiempos no estaban maduros. El soplo revolucionario solo había rozado la periferia"29.

La Asamblea Universitaria, aprobada por decreto presidencial No. 352, del 17 de marzo de $1923^{30}$, fue minada por representantes de la reacción,

28 El 20 de agosto de 1925 fue baleado Armando André, director del periódico oposicionista El Día. Las persecuciones y matanzas continuaron con los asesinatos de líderes obreros: Enrique Varona, B. Brooks, Alfredo López. Asimismo, en la región de Ciego de Ávila fueron masacrados decenas de trabajadores agrícolas oriundos de Islas Canarias.

29 Raúl Roa, "La revolución universitaria”, en Bufa Subversiva, ed. Raúl Roa (La Habana: Cultural S.A., 1935), 326.

30 La Asamblea estaba integrada por 10 profesores de cada una de las tres facultades universitarias, incluidos los decanos de las mismas, 30 estudiantes matriculados en la enseñanza oficial y que hubiesen terminado el primer año de la carrera, 30 graduados universitarios, a razón de 10 por cada facultad. Los estudiantes serían designados por la Federación de Estudiantes a propuestas de las respectivas 
al tiempo que el proyecto de autonomía universitaria, acordado por la Asamblea para presentarlo al Congreso de la República, jamás fue aprobado por las instancias gubernamentales.

Luego de varios meses sin sesionar, la Asamblea Universitaria se reunió el 12 de junio de 1925 para aceptar la renuncia del rector Enrique Hernández Cartaya, devenido Secretario de Hacienda del gobierno machadista. Mientras tanto, Mella intervendría para exigir la consumación de los puntos pendientes de la reforma institucional y docente de la Universidad. Dentro de la heterogénea Asamblea Universitaria, a la cual pertenecía, presionó para alcanzar la definitiva autonomía y la culminación de la depuración profesoral.

Pero el dictador Gerardo Machado se encargaría de ponerle el punto final a los esfuerzos del joven revolucionario, cuando de un plumazo disolvió la Asamblea Universitaria el 26 de diciembre de 1925, ilegalizó la Federación de Estudiantes y repuso a los profesores separados de sus cargos. No satisfecho, el mandatario instituyó un Directorio Estudiantil a su servicio, instrumento que le permitiría la expulsión arbitraria de Mella de la Universidad y lo obligaría, tras varias detenciones ${ }^{31}$, al exilio mexicano.

A solicitud de Julio Antonio Mella, Rubén Martínez Villena y José Zacarías Tallet, en calidad de secretario y presidente, respectivamente, inscribieron la UPJM en el registro provincial con el objetivo de protegerla de sus adversarios. Pero los días de la institución estaban contados.

Con la salida de Mella hacia México, en enero de 1926, se inició una tercera etapa de la UPJM que culminaría con su definitiva supresión. Ya no solo se le obligó a impartir sus cursos fuera de la Universidad

Asociaciones de Estudiantes. Entre sus funciones se encontraban la elección del Rector de la Universidad, acordar los proyectos que considerase procedente proponer para la reforma de los planes de estudio, y proponer al Claustro General los proyectos o reformas de los Estatutos de la Universidad.

31 A finales de 1925, Julio A. Mella y un grupo de dirigentes obreros fueron detenidos y enviados a la cárcel de La Habana, acusados de violar la Ley de Explosivos. El 5 de diciembre Mella comenzó una huelga de hambre que conmocionó a la opinión pública nacional e internacional. Luego de 18 días en ayuno y gracias al activismo del Comité Pro Libertad de Mella, fue excarcelado, pero obligado por el régimen dictatorial a exiliarse. 
Nacional, sino que su principal inspirador y guía no se encontraba en la Isla.

El quehacer político de Julio Antonio Mella en México no fue menos intenso que en $\mathrm{Cuba}^{32}$, aunque la nueva experiencia en la vecina nación le permitió madurar más de prisa su pensamiento y ejecutoria revolucionarios, al punto de descollar como líder continental. En mayo de 1926 le escribe a sus compañeros de la Universidad: "En este ambiente de agitación y experiencia revolucionarias único en la América, estoy como en una universidad de pueblos, como en un magno laboratorio de sociología aprendiendo para el obrero y campesino de Cuba, lo que nunca podría aprender allá"33.

A pesar de su militancia activa en el Partido Comunista Mexicano, del cual fue destacado redactor del órgano oficial, El Machete, su protagonismo en la Liga Antimperialista de las Américas y en el Comité Manos Fuera de Nicaragua, mantuvo los vínculos con la dirigencia y los profesores de la UPJM. También continuaron los mensajes de apoyo a todos los integrantes de la Universidad Popular, por más que la situación para la supervivencia de la institución se hacía cada vez más insostenible.

Las irregularidades no solo fueron externas, pues el plantel popular al parecer tampoco escapó de algunas insuficiencias en su ejecutoria. Fernando Sirgo y Traumont, quien fuera profesor de la UPJM, declararía posteriormente que la institución no fue tan abierta y antidogmática como habían previsto sus estatutos. Los organizadores estaban interesados sobre todo en difundir la teoría marxista y el socialismo científico, lo que provocó enfrentamientos con la mayoría de los alumnos de inclinación anarquista. Al mismo tiempo, una parte de los estudiantes, según el testigo, se interesaba más por la obtención de conocimientos de gramática, aritmética o geografía, desestimando la parte doctrinal de influjo marxista ${ }^{34}$. en Julio Antonio Mella. Documentos y artículos, 227.

34 Christine Hatzky, Julio Antonio Mella. Una biografía, 123. 
Por otra parte, el influjo de la labor divisionista del APRA también había calado en el establecimiento universitario. Una corriente que en modo alguno se circunscribía a Cuba. Mariátegui, en Perú, enfrentaba la plataforma reformista de Haya de la Torre y sus seguidores, orientada a suplantar al proletariado en la revolución antimperialista y antioligárquica. Mella, desde el exilio, fustigó el basamento doctrinal aprista y aconsejó que los representantes de la sección cubana de la Alianza Popular fueran excluidos de la UPJM.

Esta división interna facilitó en Cuba el llamado "proceso contra los comunistas", extendido a cuantas instituciones fueron tachadas de opositoras a la prórroga presidencial decretada por Machado. La prohibición gubernamental de la UPJM, en 1927, formó parte de esta reacción conservadora, y motivó una nota redactada por Julio Antonio en la que evaluaba la medida dictada por la dictadura machadista, al tiempo que enfatizaba en el alcance educativo y político de la ejecutoria del plantel en sus cuatro años de existencia:

“[...] La Universidad Popular no es una sociedad secreta insurreccional. Esto lo saben hasta los mismos policías. Pero la enseñanza de la Universidad Popular José Martí ha insurreccionado a más de una conciencia dormida y domesticada, la ha insurreccionado contra el despotismo político, contra la injusticia económica, contra la dominación extranjera, contra el "valor" de la ignorancia. La declaración de ilegalidad es un galardón más para la Universidad. En los momentos en que la fuerza bruta reina como una fórmula política, es natural que la Universidad Popular José Martí sufriera, como la Universidad y el Instituto de La Habana una "prudente" clausura" 35 .

Aún en los últimos momentos de su existencia legal, Mella convocó a la UPJM a que enviara a un representante para que asistiera al Congreso Antimperialista de Bruselas efectuado en febrero de 1927. El líder cubano participó en el cónclave europeo como delegado en representación de la Liga Nacional Campesina de México, de las secciones

35 Julio Antonio Mella, "El cuarto aniversario de la Universidad Popular José Martí", noviembre de 1927, en Julio Antonio Mella. Documentos y artículos, 327-328. 
mexicana, salvadoreña y panameña de la Liga Antimperialista de las Américas, y como parte de su Comité Continental Organizador.

Dos años después de la clausura de la UPJM era asesinado Julio Antonio Mella en la ciudad de México. Con la muerte del joven de 26 años de edad desaparecía también cualquier posibilidad de reanimar la Universidad Popular en contextos más favorables, lo cual no implicó que el papel cultural y político de esta experiencia cayera al vacío. En carta a su profesora y amiga Sarah Pascual, Mella le había señalado el significado de instituir una "universidad de revolucionarios en un país donde no había ambiente para crearlos", pero añadía que el hecho de "crearlos" no significaba que estuvieran dispuestas las condiciones para una revolución social en Cuba: "Lo importante no es pensar que vamos a realizar la revolución dentro de unos minutos, sino que estamos capacitados para aprovechar el momento histórico cuando este fatalmente llegue" 36 .

\section{Conclusiones}

El sostén ideológico marxista de la UPJM en Cuba y su orientación antimperialista y latinoamericanista, le imprimieron un carácter marcadamente radical a la propuesta educadora del líder estudiantil Julio Antonio Mella desde su establecimiento en 1923. El despliegue de la reforma universitaria favoreció la convocatoria del entonces estudiante de Derecho a su preciado proyecto de extensión universitaria, pero, al mismo tiempo, la institución obrero-estudiantil le permitió avanzar en su estrategia de lucha política; llegar allí donde no podría con las fraccionadas fuerzas de la Federación de Estudiantes ni con la espera paciente de la anhelada autonomía de la Universidad Nacional.

Mantener el funcionamiento de la UPJM era crucial, a pesar de las adversidades que la institución debió enfrentar en sus cuatro años de existencia, víctima de la reacción gubernamental y de las tendencias conservadoras y contrarreformistas hacia el interior de la Universidad

36 Julio Antonio Mella, "Carta a Sarah Pascual", México D.F., 16 de septiembre de 1926, en Julio Antonio Mella. Documentos y artículos, 257. 
de La Habana. La "niña querida” de sus sueños, como denominara Mella a la Universidad Popular, garantizaba la educación de amplios sectores, grupos y capas de la población, con niveles de instrucción variable, pero en cualquiera de los casos sujetos a los moldes estatuidos por el Estado liberal. De lo que se trataba, según el dirigente estudiantil, era sentar las bases para la transgresión ideológica del orden educativo burgués y, al efecto, la consolidación de resortes metodológicos capaces de unificar las variadas tendencias en el complejo espectro político del movimiento obrero. En ese esfuerzo habrían de articularse de manera original los lineamientos marxistas-leninistas con un nacionalismo martiano que contemplara en su formulación, no solo el independentismo, sino también el acendrado ideal antimperialista del ideólogo de la independencia.

Mella era consciente de que la labor de extensión universitaria no estaba cifrada en una pretendida transgresión inmediata del sistema capitalista, ni siquiera de la más circunstancial caída de un régimen. En su concepción política la extensión universitaria, a cargo de estudiantes y jóvenes progresistas, debía cubrir un vacío de instrucción en sectores y grupos con limitado acceso al sistema de escolarización pública, al tiempo que incluía, en el plano ideológico, una plataforma educativa y cultural contrahegemónica, capaz de sentar las bases teóricas y prácticas para el enrumbe de una revolución social en coyunturas favorables.

La ejecutoria política de la UPJM describió en su decurso una línea ascendente a tono con el quehacer revolucionario de su fundador. En la medida en que el activismo de Mella se imbricaba más con las luchas sindicales en todo el país y que su protagonismo y prestigio entre los dirigentes obreros, estudiantiles e intelectuales se incrementaban, las misiones de la Universidad Popular rebasaban la relativa quietud de los locales donde se impartían las clases nocturnas. De igual modo, la radicalidad del pensamiento y la acción del fundador del PCC y de diversas organizaciones antimperialistas condicionó también el estrechamiento del cerco gubernamental sobre la universidad proletaria. La temprana salida del recinto universitario y el posterior exilio de Mella en México marcaron puntos de definición críticos en el desarrollo de la actividad de la UPJM, clausurada definitivamente en 1927. 


\section{BiBliografía}

\section{Fuentes primarias}

\section{Archivo}

Archivo Histórico del Instituto de Historia de Cuba (AHIHC). La Habana, Cuba. 1/2: PE 2.1/1/1/1, Fondo Primer Partido Marxista-Leninista.

\section{Publicaciones periódicas}

Justicia, La Habana, 13 de enero de 1923.

Boletín de la Universidad Popular José Martí, La Habana, 13 de julio de 1924.

El Heraldo, La Habana, 26 de noviembre de 1924.

Escritos de Julio Antonio Mella

"Estatutos de la Universidad Popular José Martí", 3 de noviembre de 1923. En Mella. Textos escogidos, compilado por Julio César Guanche, t. I. La Habana: Ediciones La Memoria, Centro Cultural Pablo de la Torriente Brau, 2003, 104-105.

"Carta a Araoz Alfaro", Camagüey, 3 de enero de 1924. En Hasta que llegue el tiempo, editado por Adys Cupull y Froilán González. La Habana: Editora Política, 2002, 197.

"A los alumnos de la Universidad Popular y al pueblo de Cuba", 3 de agosto de 1924. En Julio Antonio Mella. Documentos y artículos, editado por el Instituto de Historia del Movimiento Comunista y la Revolución Socialista de Cuba. La Habana: Editorial de Ciencias Sociales, 1975, 100-101.

"Carta al representante del Perú", mayo de 1924. En Mella. 100 años, editado por Ana Cairo, Vol. II. Santiago de Cuba: Editorial Oriente, $2003,175$.

"Hablando con Julio Antonio Mella sobre la revolución universitaria", La Habana, 23 de noviembre de 1924. En Julio Antonio Mella. Documentos y artículos, editado por el Instituto de Historia del Movimiento Comunista y la Revolución Socialista de Cuba. La Habana: Editorial de Ciencias Sociales, 1975, 133.

"Mensaje a los compañeros de la Universidad Popular", México, marzo de 1926. En Julio Antonio Mella. Documentos y artículos, editado por el Instituto de Historia del Movimiento Comunista y la Revolución Socialista de Cuba. La Habana: Editorial de Ciencias Sociales, 1975, 227. 
“Carta a Sarah Pascual”, México D.F., 16 de septiembre de 1926. En Julio Antonio Mella. Documentos y artículos, editado por el Instituto de Historia del Movimiento Comunista y la Revolución Socialista de Cuba. La Habana: Editorial de Ciencias Sociales, 1975, 256-257

"El cuarto aniversario de la Universidad Popular José Marti", noviembre de 1927. En Julio Antonio Mella. Documentos y artículos, editado por el Instituto de Historia del Movimiento Comunista y la Revolución Socialista de Cuba. La Habana: Editorial de Ciencias Sociales, 1975, 326-328.

"El concepto socialista de la reforma universitaria". El Tren Blindado, México (s/f), Archivo Histórico del Instituto de Historia de Cuba (AHIHC), Fondo Primer Partido Marxista-Leninista, 1/2: PE 2.2/7/63. A1/1-15.

\section{Otros}

"Plan de estudios y profesores de la Universidad Popular José Martí".

En Mella. 100 años, editado por Ana Cairo, Vol. I. Santiago de Cuba: Editorial Oriente, 2003, 51-52.

Hernández, Eusebio y Domingo Ramos. "Homicultura”. Boletín de Sanidad y Beneficencia T. VI, No. 1-6 (La Habana, 1911).

\section{Fuentes secundarias}

Aguirre, Sergio. "Un toque de clarín en 1923”. Bohemia, La Habana, 19 de octubre de 1973.

Cabrera, Olga. Los que viven por sus manos. La Habana: Editorial de Ciencias Sociales, 1985.

Cairo, Ana (ed.). Mella. 100 años, 2 vols. Santiago de Cuba: Editorial Oriente, 2003.

Cupull, Adys y Froilán González. Hasta que llegue el tiempo. La Habana: Editora Política, 2002.

Dumpierre, Erasmo. Julio Antonio Mella. Biografía. La Habana: Editorial Orbe, 1975.

Guanche, Julio César. “julio Antonio, qué pasa en Cuba?”. En Mella. 100 años, editado por Ana Cairo, Vol. II. Santiago de Cuba: Editorial Oriente, 2003.

Hatzky, Christine. Julio Antonio Mella. Una biografía. Santiago de Cuba: Editorial Oriente, 2008. 
Ibarra Cuesta, Jorge. Cuba 1898-1921. Partidos políticos y clases sociales. La Habana: Editorial Ciencias Sociales, 1992.

Kohan, Néstor. "Mella, reforma universitaria y revolución”. En Mella. 100 años, editado por Ana Cairo, Vol. II. Santiago de Cuba: Editorial Oriente, 2003.

Le Riverend, Julio. La república, dependencia y revolución. La Habana: Editora Universitaria, 1966.

Moreno Martínez, Pedro Luis. "Haciendo memoria: las Universidades Populares en España”. Tabanque. Revista Pedagógica No. 19 (2005): 21-40.

Padrón, Pedro Luis. Julio Antonio Mella y el movimiento obrero. La Habana: Editorial de Ciencias Sociales, 1980.

Pascual, Sarah. Julio Antonio Mella. Síntesis de su vida. La Habana: Universidad de La Habana. Instituto Julio Antonio Mella, 1971.

Roa, Raúl. Bufa Subversiva. La Habana: Cultural S.A., 1935.

Roa, Raúl. "Una semilla en el surco de fuego". En Órbita de Rubén Martínez Villena, selección de Roberto Fernández Retamar. La Habana: Colección Órbita, UNEAC, 1964.

Para citar este artículo: Cordoví Núñez, Yoel. "La Universidad Popular José Martí en la órbita del pensamiento político de Julio Antonio Mella. Cuba, 1923-1927", Historia Caribe Vol. XVI No. 38 (Enero-Junio 2021): 107-136. DOI: https://doi.org/10.15648/hc.38.2021.2813 